Draft version February 5, 2008

Preprint typeset using IATEX style emulateapj v. 6/22/04

\title{
ENVIRONMENT AND THE COSMIC EVOLUTION OF STAR FORMATION
}

\author{
Ravi K. Sheth, ${ }^{1}$ Raul Jimenez, ${ }^{1,2}$ Ben Panter $^{3}$, and Alan F. Heavens ${ }^{4}$
}

Draft version February 5, 2008

\begin{abstract}
We present a mark correlation analysis of the galaxies in the Sloan Digital Sky Survey using weights provided by MOPED. The large size of the sample permits statistically significant statements about how galaxies with different metallicities and star formation histories are spatially correlated. Massive objects formed a larger fraction of their stars at higher redshifts and over shorter timescales than did less massive objects (sometimes called down-sizing). We find that those galaxies which dominated the cosmic star formation at $z \approx 3$ are predominantly in clusters today, whereas galaxies which dominate the star formation at $z \approx 0$ inhabit substantially lower mass objects in less dense regions today. Hence, our results indicate that star formation and chemical enrichment occured first in the denser regions of the Universe, and moved to less dense regions at later times.
\end{abstract}

Subject headings: stellar populations - large scale structure of universe

\section{INTRODUCTION}

There has been substantial recent progress in the development of methods which determine the star formation and chemical composition histories of galaxies from the integrated spectra of their stellar populations. The traditional approach has been to determine the instantaneous star formation rate or metallicity from certain features in the spectrum of a galaxy. However, several recent algorithms (Heavens et al. 2000; Sodre et al. 2005; Mathis et al. 2006; Ocvirk et al. 2006), have been developed which use the entire spectrum to infer the entire star formation history and the evolution of the chemical composition of the object, rather than simply the instantaneous values of these quantities. One such method, MOPED (Heavens et al. 2000), has been used to determine the star formation histories and metallicities of galaxies drawn from the Sloan Digital Sky Survey (Panter et al. 2003; Heavens et al. 2004; Panter et al. 2004; Jimenez et al. 2005; Panter et al. 2006a b).

There has also been significant progress in quantifying how galaxies are distributed on large scales, and using this to constrain cosmological parameters (Cole et al. 2005: Seliak et al. 2005). In such analyses of galaxy clustering, it is common to treat galaxies as points, ignoring the fact that galaxies have different luminosities, colors, masses, star formation histories, metallicities, etc. However, as a result of improvements in detector technology, and in the algorithms such as MOPED with which the new data is analyzed, many such galaxy attributes are now sufficiently reliably measured that one can use them as weights when studying the clustering of galaxies. Thus, one can now study the clustering of luminosity, color, star formation rate, etc. Mark statistics

\footnotetext{
1 Dept. of Physics and Astronomy, University of Pennsylvania, 209 South 33rd St, Philadelphia, PA 19104, U.S.A.; shethrk,raulj@physics.upenn.edu

2 The Observatories of the Carnegie Institution, 813 Santa Barbara St., Pasadena, CA 91101, U.S.A.

3 Max Planck Institut für Astrophysik, Karl Schwarzschild Str. 1, Garching bei München, D-85748, Germany; bdp@mpagarching.mpg.de

4 Institute for Astronomy, University of Edinburgh, Blackford Hill, Edinburgh, EH9-3HJ, Scotland; afh@roe.ac.uk
}

(Stovan \& Stovan 1994; Beisbart \& Kerscher 2000) provide a useful framework for describing attribute-weighted clustering. Moreover, they provide sensitive probes of how the properties of galaxies correlate with their environments (Sheth 2005). In this respect, mark statistics provide a useful link between the large-scale structures which galaxies trace, and the properties of those galaxies. They have recently been used to measure the clustering of luminosity and color in the SDSS (Skibba et al. 2006).

In this Letter, we use mark correlations to demonstrate the wealth of information which the MOPED-determined histories provide. We show that a mark correlation analysis, using MOPED attributes as marks, allows one to study how the star formation histories of objects are correlated with their present-day environments. Our main finding is that the majority of close $(<2 \mathrm{Mpc})$ galaxy pairs today are made up of objects which formed the largest fraction of their stars at $z \approx 3$, whereas those which formed stars at an above average rate within the last Gyr or so tend to be in less clustered environments today. In hierarchical models, overdense regions today were overdense in the past. Thus, our results indicate that star formation at high-redshift occured in dense regions, whereas it only occurs in substantially less dense regions today. A similar study of the metallicity shows that the close pairs which formed a larger than average fraction of their stars at $z \approx 3$ also have above average metallicities, but that there is no correlation between metallicity and environment for stars formed more recently. Where necessary, we assume a flat $\Lambda$ CDM model with $\Omega_{0}=0.27$ and $H_{0}=71 \mathrm{~km} \mathrm{~s}^{-1} \mathrm{Mpc}^{-1}$ (Spergel et al. 2006).

\section{MEASUREMENTS IN THE SDSS}

As described in Panter et al. (2006a b) , the MOPED algorithm has been used to extract star formation and metallicity histories of a magnitude limited sample $\left(15.0 \leq m_{r} \leq 17.77\right)$ of about 300,000 galaxies drawn from the Third Data Release of the Sloan Digital Sky Survey (SDSS DR3; Abazaiian et al. (2005)). We measured mark correlations, using MOPED attributes as the marks, in two smaller volume-limited catalogs extracted 


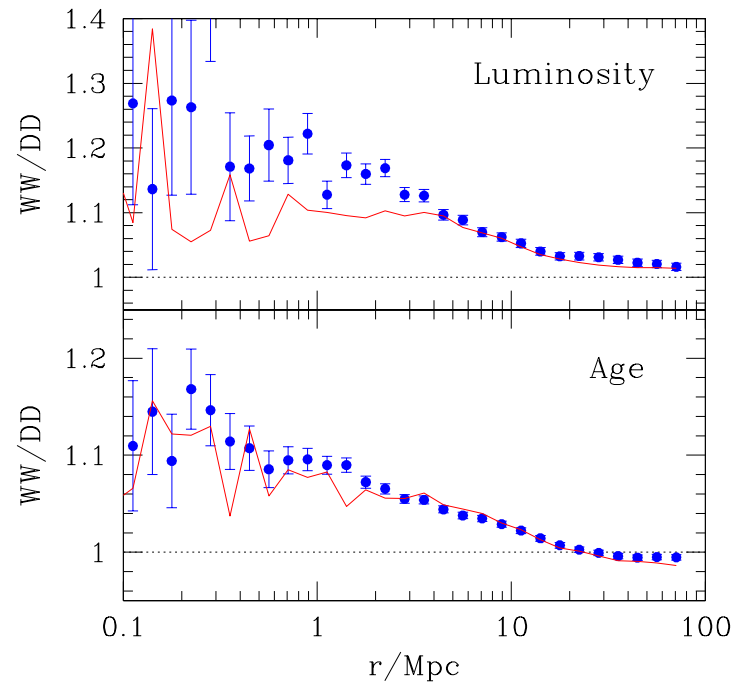

FIG. 1.- Mark correlations in the two volume limited catalogs described in the text; symbols with error bars show results for the fainter sample, and lines without error bars are for the more luminous sample. Close pairs tend to be more luminous (top panel) and to host older stellar populations (bottom) than average.

from the SDSS DR3. To facilitate future halo-model based interpretations of our measurements, these catalogs were chosen to approximately correspond to those studied by Zehavi et al. (2005) and Skibba et al. (2006): a brighter catalog which spans $M_{r}<-21.5$ for which $0.02<z<0.135$, and one which includes fainter objects, $M_{r}<-20$, and so spans a smaller redshift range: $0.02<z<0.071$ (the associated apparent magnitude limit is conservative: $\left.m_{r} \leq 17.5\right)$.

Figure 1 shows the mark correlations in the two catalogs when $r$-band luminosity (top) and the MOPEDinferred mass-weighted age (bottom) are the marks (symbols with error bars show results for the fainter sample, and lines without error bars are for the brighter sample). So that the weights are dimensionless, the weight of each galaxy is normalized by the mean value for the population. The notation WW/DD indicates that the mark statistic is the ratio of weighted pair counts to unweighted pair counts. (In this notation, the traditional unweighted correlation function would be $\mathrm{DD} / \mathrm{RR}$, where $\mathrm{RR}$ is the number of unweighted pair counts in a random distribution.) Symbols with error bars show results for the fainter catalog, and lines without error bars are for the more luminous sample - we use a similar convention in all the figures which follow. Error bars were estimated using the analytic expressions given in Sheth et al. (2006), which Skibba et al. (2006) have shown are similar to those from a jackknife analysis; they are similar for the two samples. The results of Skibba et al. also show that, while using redshift- rather than real-space distances tends to make WW/DD closer to unity on small scales, this is not a severe effect.

The top panel shows that close pairs of galaxies tend to be more luminous than average, consistent with previous mark correlation analyses of SDSS galaxies (Skibba et al. 2006). Halo-model based analyses of the clustering

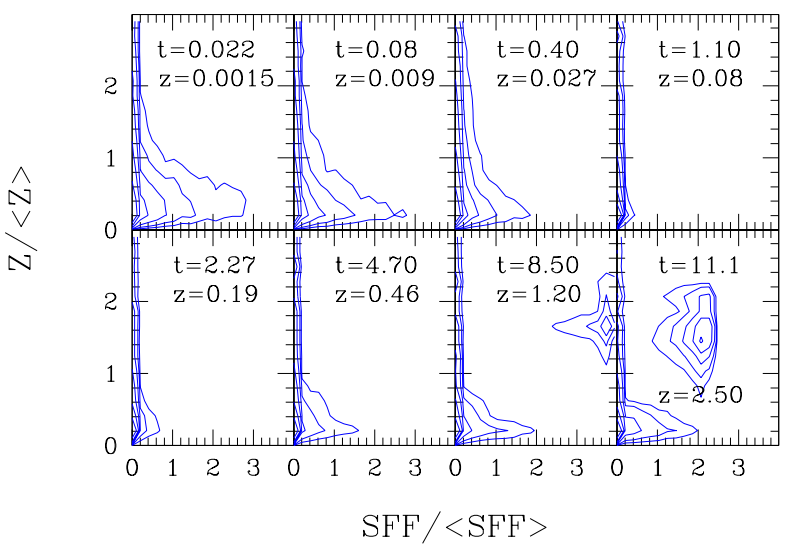

FIG. 2.- Joint distribution of star-formation fraction and metallicity in the volume limited catalog with $M_{r}<-20$; Different panels show results for different lookback times, $t$ in Gyrs, and associated redshifts, $z$. In all panels, the marks have been normalized by the mean mark in the bin.

of SDSS galaxies indicate that, on scales smaller than $1 \mathrm{Mpc}$, the pair counts are dominated by galaxies in massive haloes (Zehavi et al. 2005). Thus, the top panel shows that galaxies in massive haloes are over-luminous.

The bottom panel shows that, in addition to being more luminous than average, close pairs tend to have older than average stellar populations. This suggests that the most massive halos host the oldest stellar populations. The scale dependence in the bottom panel is also qualitatively similar to that seen in semi-analytic galaxy formation models (Sheth 2005; Sheth et al. 2006). In the models, this happens because close pairs are dominated by galaxies in clusters, and cluster galaxies host the oldest stars. This is consistent with the halo-model interpretation mentioned above.

Figure 1uses marks which are the result of integrating over the entire star formation history of each object. One of the great virtues of the MOPED analysis is that it returns not just the mass-weighted age at the present time, but an estimate of the entire star formation history of an object. Thus, for each object, we have constructed estimates of the fraction of the current stellar mass which formed in each of eight bins in lookback time.

Figure 2 shows the joint distribution of star formation fraction and metallicity in the fainter catalog, for each of the lookback time bins. In each panel, the marks have been normalized by the mean value in the bin. For instance, at the two largest lookback times, $\left(\langle\mathrm{SFF}\rangle,\left\langle\mathrm{Z} / \mathrm{Z}_{\odot}\right\rangle\right)=(0.25,0.65)$ and $(0.43,0.77)$. These numbers are $(0.25,0.70)$ and $(0.55,0.89)$ in the more luminous catalog. The differences indicate that massive objects formed a larger fraction of their stars at higher redshifts, on a shorter timescale, than lower mass objects - a point made by Heavens et al. (2004).

In all panels there is a population of objects which have small star formation fractions but a large range of metallicities, and a population which has small metallicities but a tail which extends to large star formation fractions. However, at large lookback times, there is an additional population which has above average metal- 


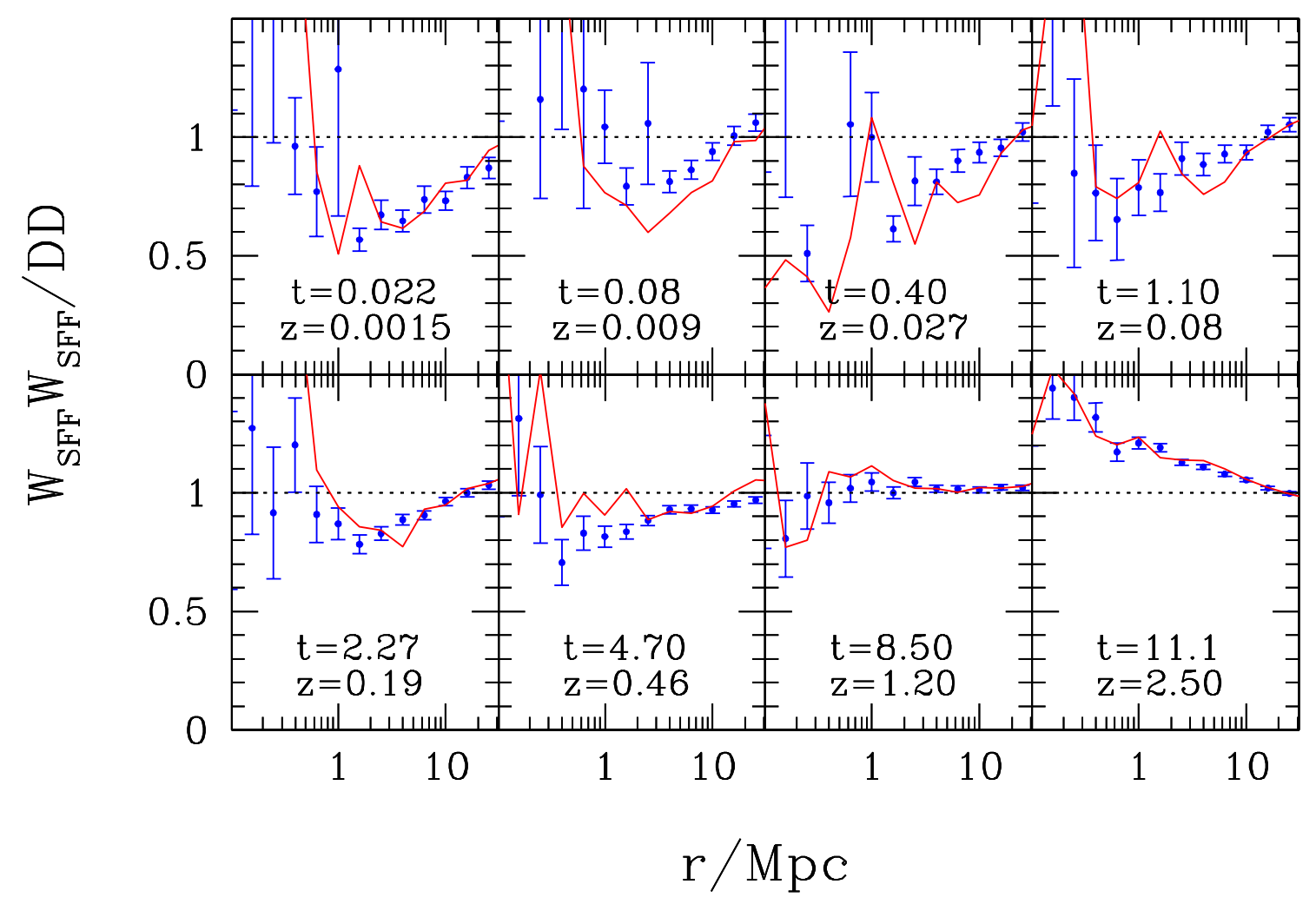

FIG. 3.- Mark correlation functions, with star-formation fraction as the mark, in the two volume limited catalogs described in the text. Symbols with error bars show results for the fainter sample; lines without error bars represent the more luminous sample. Panels show results for different lookback times, $t$ in Gyrs, with associated formation redshifts, $z$. In both catalogs: close pairs today had higher than average star formation fractions at $z=2.5$, average star formation fractions at $z=1$, and lower than average star formation fractions more recently. The anti-correlation between star formation fraction and environment persists up to lookback times of 5 Gyrs.

licity and star formation fraction. For the two largest lookback times, this population comprises $24 \%$ and $39 \%$ of the galaxies in the fainter catalog $(26 \%$ and $51 \%$ in the brighter catalog). Where are these objects now?

Figure 3 shows a mark correlation analysis of the star formation fraction at these eight lookback times. The bottom right panel shows that close pairs today had larger than average star formation fractions 11 Gyrs ago. Comparison with the other panels indicates that these pairs had average star formation fractions at redshifts of order unity, and smaller than average star formation fractions more recently. Thus, our analysis provides graphic evidence that the objects which underwent vigorous star formation at the highest redshifts are currently in clusters, where the current star formation rate is smaller than average.

Figure 4 shows a similar analysis when metallicity, $Z / Z_{\odot}$, is the mark. The bottom right panel shows that the close pairs which had above average star formation fractions at $z=2.5$ also tend to have above average metallicities. There are no clear correlations with environment in the other panels, except, possibly, at the smallest lookback times (top left), where there is an enhancement on scales where the pair counts are dominated by galaxy groups. Thus, our measurements indicate that the stellar populations of the most massive halos are old and metal rich. The population of objects which had above average star formation fractions and metallicities at large lookback times (c.f. Figure 2) are today in clusters.

\section{CONCLUSIONS}

A mark correlation analysis of SDSS galaxies using MOPED-derived ages, metallicities and star formation histories shows that close pairs tend to host stellar populations which are older than average (Fig. 11). Close pairs also tend to have formed a larger fraction of their stars at $z \approx 3$ than average, but the star forming fraction at $z<1$ of such pairs is below average (Fig. 13). The objects which were forming stars at $z \approx 3$ have above average metallicities (Fig. (4). These trends do not depend significantly on the mean luminosity of the sample, so they are approximately independent of stellar mass.

Close pairs are dominated by galaxies in massive halos. Hence, our results indicate that galaxies which formed the bulk of their stars at high redshift are today in clusters, in which there is little ongoing star formation. Since clusters formed from overdense regions in the early Universe, our results imply that cosmic star formation has moved from dense to ever less dense regions. This is qualitatively consistent with the findings of Poggianti et al. (2006). However, whereas Poggianti et al. measure instantaneous star formation rates in cluster galaxies identified over a wide range of redshifts (the SDSS at $z \sim 0$, 


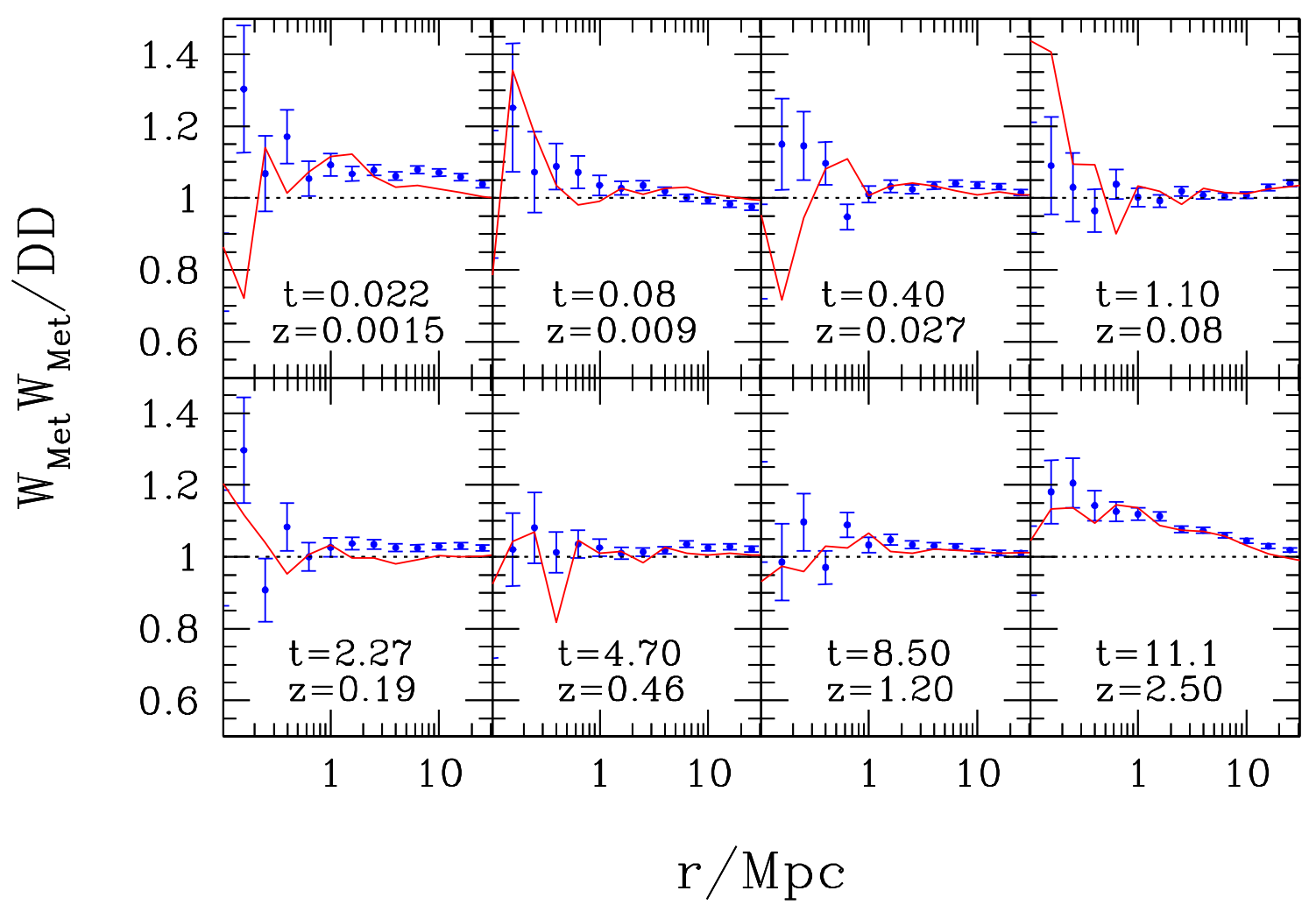

FIG. 4.- Mark correlation functions with metallicity as the mark in the two volume limited catalogs described in the text. Symbols with error bars show results for the fainter sample, and lines without error bars are for the more luminous sample. Panels show results for different lookback times. Close pairs which formed their stars 11 Gyrs ago tend to have metallicities which are above average for that epoch (bottom right). At smaller lookback times there is little correlation between metallicity and present day environment except very recently (top left) where most of the enrichment is taking place in galaxy groups.

and the ESO Distant Cluster Survey for $0.4 \leq z \leq 0.8$ ), our method uses the spectra of the local galaxy population to infer the entire cosmic star formation history. In particular, it does not require classification of the galaxies into 'cluster' and 'field' populations, nor does it require acquisition of a galaxy sample which spans a wide redshift range. It is remarkable that these two very different methods agree.

For similar reasons, the top left panel of Fig. 3 may be compared with recent studies of the dependence of current star formation on environment (Gomez et al. 2003; Balogh et al. 2004; Mateus et al. 2006). We all find smaller star formation rates in dense regions today. Note, however, that our analysis is not restricted to the current epoch - it covers 11 Gyrs in lookback time. Halo model interpretations of our measurements will help determine if the environment plays a crucial role in regulating star formation.

RKS is supported by NASA-ATP NAG-13720 and by the NSF under grant AST-0520647. RJ is supported by NSF grants AST-0408698 and PIRE-0507768, and NASA grant NNG05GG01G. BDP is supported by the Alexander von Humboldt Foundation, the Federal Ministry of Education and Research, and the Programme for Investment in the Future (ZIP) of the German Government.

Funding for the SDSS has been provided by the Alfred P. Sloan Foundation, the Participating Institutions, the National Science Foundation, the U.S. Department of Energy, NASA, the Japanese Monbukagakusho, and the Max Planck Society.

\section{REFERENCES}

Abazajian, K., et al. (the SDSS collaboration) 2005, AJ, 129, 1755 Balogh, M., et al., 2004, MNRAS, 348, 1355

Beisbart C., \& Kerscher, M. 2000, ApJ, 545, 6

Cole, S., et al., (the 2dF collaboration) 2005, MNRAS, 362, 505

Gomez, P. L., et al., 2003, ApJ, 584, 210

Heavens, A. F., Jimenez, R., \& Lahav, O. 2000, MNRAS, 317, 965

Heavens, A., Panter, B., Jimenez, R., \& Dunlop, J. 2004, Nature, 428,625

Jimenez, R., Panter, B., Heavens, A. F., \& Verde, L. 2005, MNRAS, 356,495

Mateus, A., Sodre, L., Cid Fernandes. R., \& Stasinska, G. 2006 MNRAS, submitted (astro-ph/0604063)
Mathis, H., Charlot, S., \& Brinchmann, J., 2006, MNRAS, 365, 385

Ocvirk, P., Pichon, C., Lançon, A., \& Thiébaut, E., 2006, MNRAS, 365, 46

Panter, B., Heavens, A. F., \& Jimenez, R. 2003, MNRAS, 343, 1145

Panter, B., Heavens, A. F., \& Jimenez, R. 2004, MNRAS, 355, 764

Panter, B., Jimenez, R., Heavens, A. F., \& Charlot, S. 2006, MNRAS, in preparation

Panter, B., Jimenez, R., \& Heavens, A. F. 2006, MNRAS, in preparation

Poggianti, B. M., et al., 2006, ApJ, in press (astro-ph/0512391)

Seljak, U., et al., 2005, Phys. Rev. D, 71, 103515 
Sheth, R. K. 2005, MNRAS, 364, 796

Sheth, R. K.. Connollv, A. J., \& Skibba, R. 2006, MNRAS, submitted (astro-ph/0511773)

Skibba, R., Sneth, K. K.. Connollv. A. J., \& Scranton, R. 2006, MNRAS, in press (astro-ph/0511773

Sodre, L., Cid Fernandes. K. Niateus, A., Stasinska, G., Gomes, J. M., 2005, astro-ph/0506420
Spergel, D. N., et al. 2006, ApJ, submitted (astro-ph/0603449

Stoyan D., \& Stoyan H., 1994, Fractals, Random shapes, and Point

Fields. Wiley \& Sons, Chichester

Zehavi, I., et al., 2005, ApJ, 630, 1 